\title{
ATENCIÓN A LA DIVERSIDAD DE CALIDAD
}

\author{
ATTENTION TO THE QUALITY DIVERSITY
}

\author{
Jesús Miguel Muñoz Cantero*, María Paula Ríos de Deus** \\ y Eva María Espiñeira Bellón*** \\ Unividasidade da Coruña
}

\section{RESUMEN}

Existen numerosos trabajos sobre temas de calidad educativa. Parece necesario reflexionar sobre aspectos que, en principio, no afectan de manera directa a toda la comunidad educativa: la atención a la diversidad y las necesidades educativas especiales (NEE). Elaboramos unos instrumentos para identificar aquellos elementos que pudieran ser identificativos de una atención a la diversidad de calidad y para que el profesorado, familia, inspección educativa, ... nos diesen su opinión sobre ello. Partiendo de lo anterior:

a) Se enfocó hacia la atención a la diversidad en los centros educativos de educación secundaria integrando aspectos referentes a la planificación, organización, materiales, ...

b) Se estructura de forma similar al modelo CIPP de Stufflebeam (Context, Input, Process, Product) para analizar los aspectos contextuales, de entrada y recursos que condicionan una atención de calidad para evaluar la utilidad y calidad de los resultados obtenidos.

Palabras clave: Educación, calidad, diversidad.

\begin{abstract}
There are many works about quality education themes. It seems necessary think about some aspects that, at the beginning do not affect in a direct way to all educative community: the attention to the diversity and special educative necessities. We elaborated an instrument to identify those elements which could be identificatives of an attention to the quality diversify and for teachers, family, educative inspection, ... give us their opinion about them.

* Jesús Miguel Muñoz Cantero, Doctor en Pedagogía, es Profesor titular de la Universidade da Coruña. Líneas de investigación en Métodos de Investigación, Evaluación y Calidad, Atención a la Diversidad.

** María Paula Ríos de Deus, es Licenciada en Pedagogía y en Psicopedagogía, desarrolla sus funciones profesionales en la Axencia para a Calidade do Sistema Universitario de Galicia (ACSUG). Santiago de Compostela. Líneas de investigación en Métodos de Investigación, Evaluación y Calidad, Atención a la Diversidad.

*** Eva María Espiñeira Bellón, Licenciada en Psicopedagogía. Lugar de trabajo: Unidad Técnica de Evaluación (UTE). Rectorado-Universidade da Coruña. Líneas de investigación en Métodos de Investigación, Evaluación y Calidad, Atención a la Diversidad.
\end{abstract}


a) It was focused towards the attention of diversity in the educative centers of primary and secondary education including aspects which make reference to planification, organisation and materials.

b) It is structured in a similar way to the model CIPP of Stufflebeam (Context, Input, Process, Product) to analyse the contextual aspects of entry and and materials which cause an quality attention and evaluate the utility and quality of got results.

Main words: education, quality, diversity

\section{Introducción}

Existe un interés creciente por el término "calidad", al que continuamente se hace referencia en diferentes ámbitos de nuestra vida, aunque signifique cosas diferentes para diferentes personas e, incluso, para una misma persona en diferentes momentos y situaciones dependiendo de cada una de las teorías y definiciones que sobre él encontramos, de los objetivos, intereses, necesidades y perspectivas que se contemplan en cada caso.

La concepción de la calidad educativa es uno de los temas más actuales en la nueva Ley de ordenación universitaria (LOU) y en la propuesta del gobierno de la mejora de la calidad en secundaria. Dicho concepto, se asocia, generalmente, con múltiples acepciones: atención a las necesidades del alumnado, optimización del proceso de enseñanza-aprendizaje, adecuación de las relaciones interpersonales, construcción activa de conocimientos, ... fomentando el cambio de la escuela ordinaria hacia una escuela integradora cuya meta es alcanzar una educación de calidad para todos y todas. Surge así la necesidad de realizar una evaluación de los centros educativos con la finalidad de desarrollar planes eficaces que posibiliten la mejora. Según De la Orden (1989: 8) "es un hecho comúnmente admitido" que debe abarcar todos los componentes institucionales.

\section{Perspectiva histórica de la integración}

En España, a partir del curso 85/86 se comienza el programa de integración escolar del alumnado con necesidades educativas especiales (NEE) de forma planificada, sistemática, continua y completa como respuesta a los intentos de experiencias aisladas y dispersas de integración realizados hasta entonces en diferentes centros y cursos (López Sepúlveda, 1988).

Podemos afirmar, al igual que García Pastor (1993) que la integración es un proceso dinámico y cambiante, cuyo objetivo central es encontrar la mejor situación educativa para que el alumnado desarrolle al máximo sus posibilidades, variando según sus necesidades y el tipo de respuesta que los centros pueden proporcionar (Gráfico 1).

En el gráfico 2 vemos la evolución contrastada de la educación especial y de lo que supone en la actualidad.

A modo de síntesis, la educación especial es una acción educativa de fines equiparables a los de la educación general, pero que actúa en base a unos recursos específicos puestos a disposición de cualquier persona; en unos casos se necesitará de forma temporal y en otros de forma continua y permanente. Las situaciones de diversidad educativa en términos de recursos y de procesos para dar respuesta a estas necesidades son las notas diferenciadoras de 


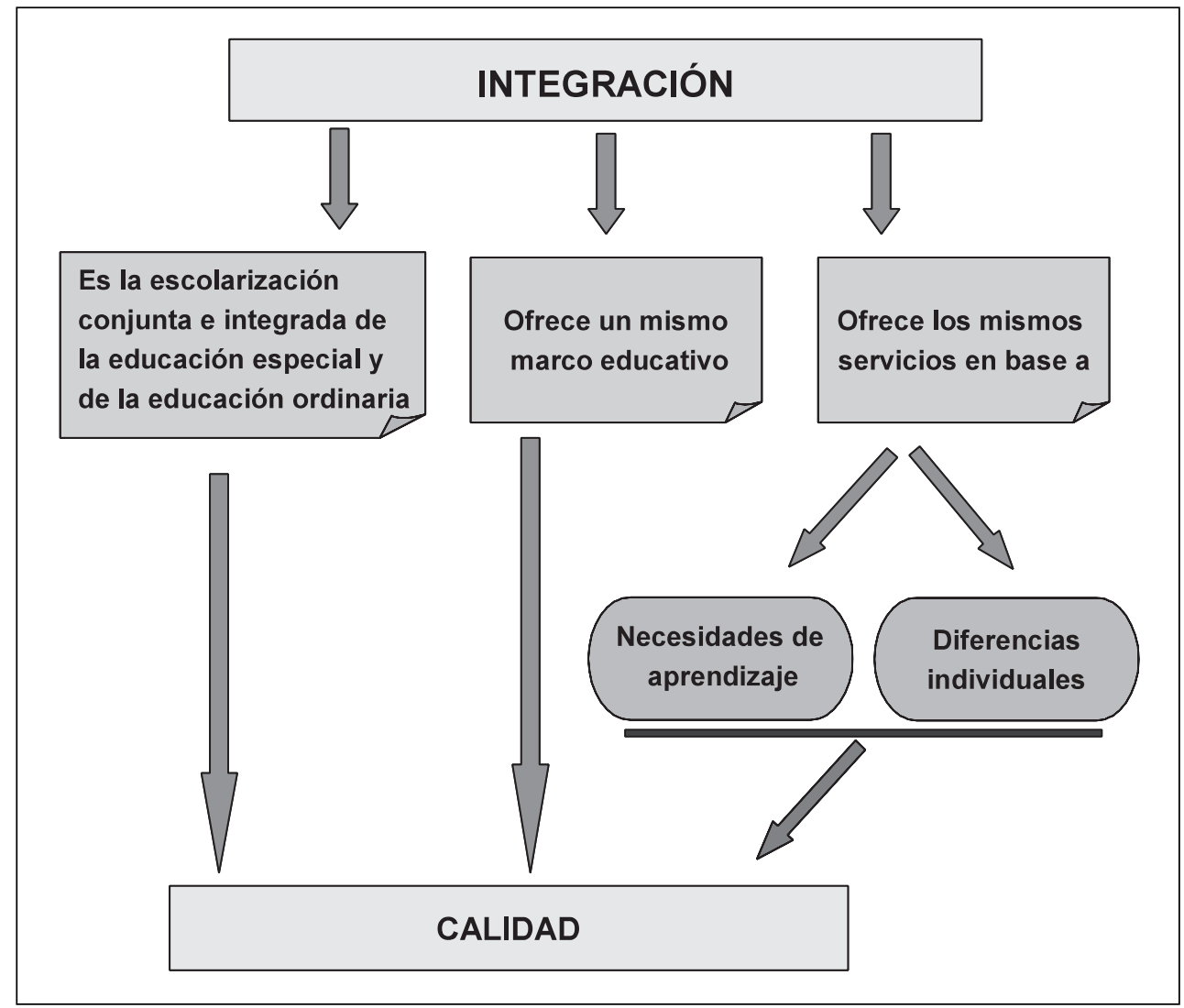

GRÁFICO 1.

Definición de integración.

la educación especial. Estos fines son compartidos por la educación ordinaria y la educación especial para poder llegar al desarrollo integral de la persona. Fines, recursos y procesos que serán aspectos clave en el desarrollo dimensional de la calidad en la atención a la diversidad. En el gráfico 3 señalamos las características que, a nuestro entender, debería cumplir una educación especial de calidad. Entendemos que cada uno de los elementos que se proponen tienen que estar interactuando, constantemente, unos con otros, con una directa atención de diversidad en sus recursos y en sus procesos.

\section{Factores que determinan una integración de calidad en centros educativos}

Cuando hablamos de integración escolar, como afirma Muñoz Cadavid (2000: 33) "es necesario realizar un análisis acerca de todas aquellas variables que en el contexto educati- 


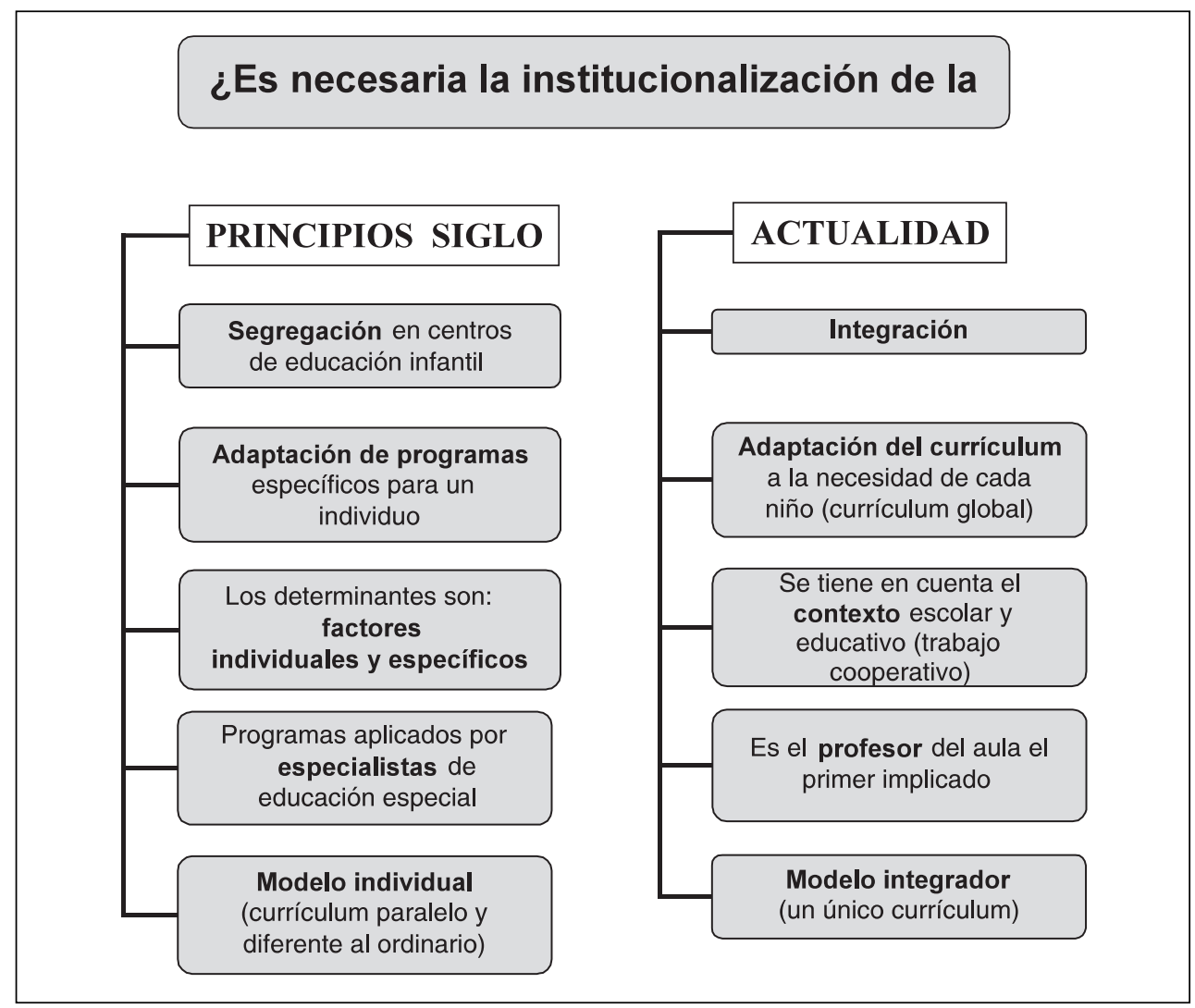

\section{GRÁFICO 2. \\ Necesidad de la institucionalización de la enseñanza.}

vo pueden facilitar o dificultar dicha integración". En ello ha de poseer una importancia esencial la evaluación para analizar su calidad y promover la mejora definiendo para ello, diversos indicadores ${ }^{1}$.

Siguiendo el modelo de evaluación presentado por De Miguel (1994) y las directrices del modelo CIPP de Stufflebeam (Context, Input, Process, Product) delimitaremos, a partir de ahora, las dimensiones o aspectos de los centros educativos que están directamente relacionados con los objetivos de la evaluación. Nos iremos centrando en cada una de las diferentes dimensiones; definiéndolas y determinando los aspectos más esenciales a partir de

${ }^{1}$ Entenderemos por indicadores, tal como lo hace De la Orden (1995) como una variable significativa, frecuentemente cuantitativa, que tienen carácter normativo.

De Miguel (1994) nos advierte que los indicadores señalan la presencia o ausencia de un hecho y que frecuentemente tienen una expresión numérica que recibe el nombre de índice. 


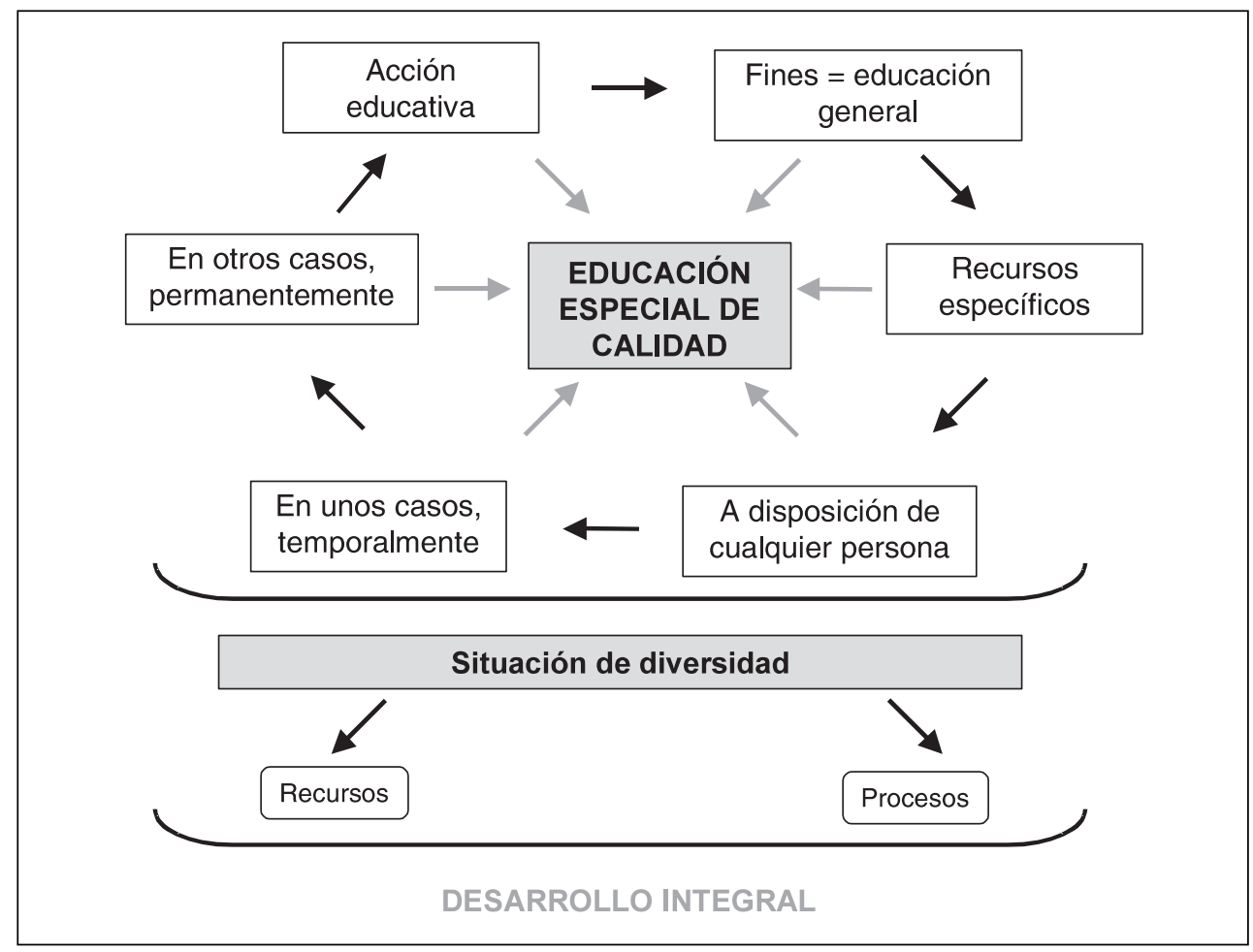

GRÁFICO 3.

Educación especial de calidad.

los cuales sería necesario recoger información posterior a través de indicadores, tal y como reflejamos en el gráfico 4.

El contexto es una fuente de demandas y una oferta de posibilidades en el que está inmerso el alumnado, la familia y el centro. Está determinado por una serie de características: geográficas, sociales, económicas, y culturales que son las que debemos tener presentes para la recogida de información, a partir de las cuales se elaborarán los indicadores y se obtendrán los datos característicos del centro así como aquellos que necesitan ser mejorados.

En el instrumento elaborado para realizar un análisis de aquellos elementos que pudieran ser identificativos de una atención a la diversidad de calidad y para que el profesorado nos diese una opinión acerca de cada una de las cuestiones a valorar, se introdujeron 10 indicadores para recoger cada uno de los aspectos a valorar en el contexto. Además, en cada apartado, se valoran también los principales puntos débiles, fuertes y propuestas de mejora que propondrían para favorecer una mayor calidad en la atención a la diversidad, en lo cual se ofrece la posibilidad de dar una respuesta más cualitativa.

Las metas y objetivos que el centro tenga respecto a la atención a la diversidad deben ser el marco de referencia de las decisiones y actividades que los centros deben considerar a 


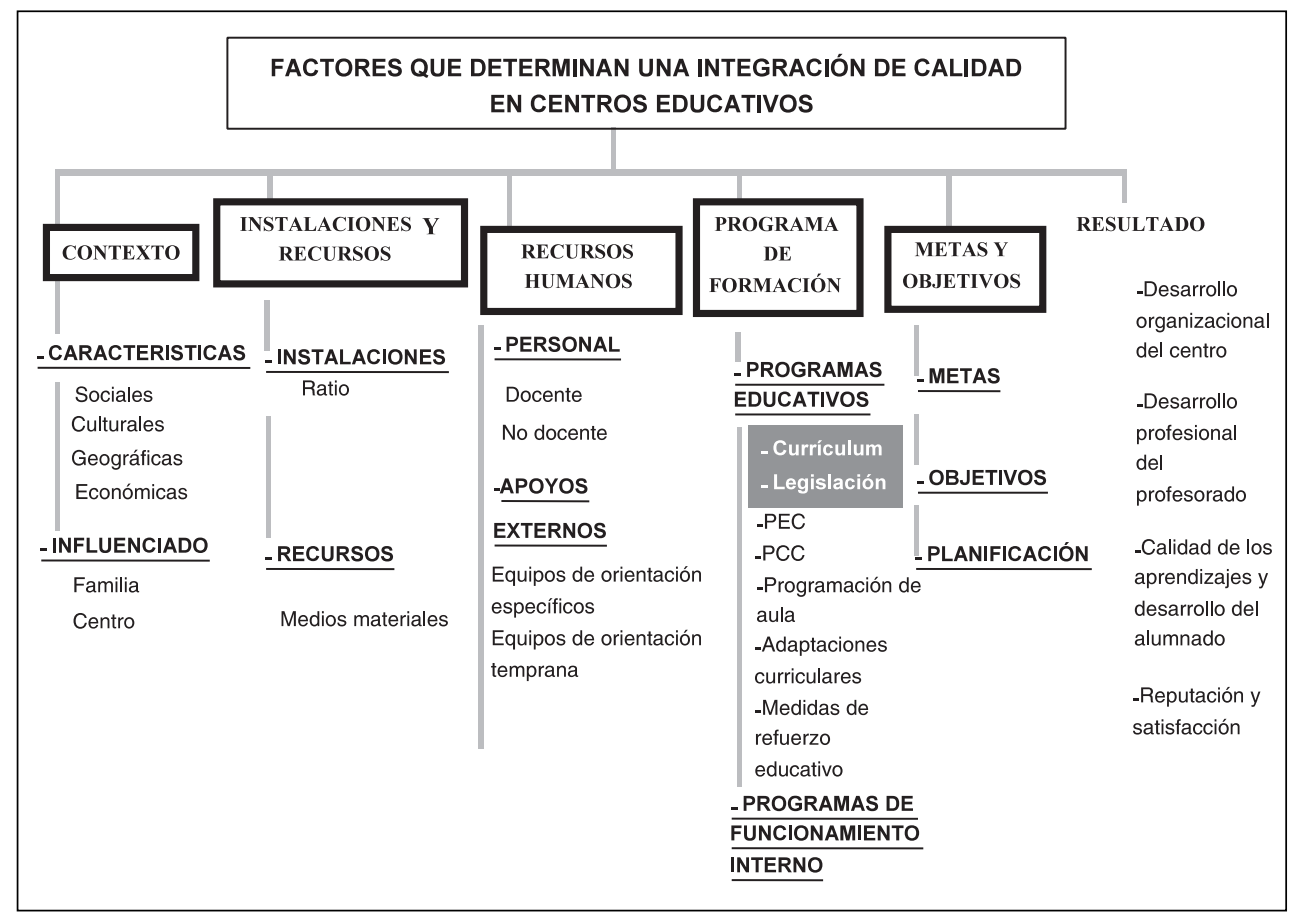

GRÁFICO 4.

Factores que determinan una integración de calidad en centros educativos.

la hora de establecer todo marco de actuación. Sería positivo, a la hora de definirlos, tener en cuenta factores como los recursos humanos, la estructura organizativa, la tipología de centro en relación con su contexto, las infraestructuras, la viabilidad de los objetivos propuestos, ... La planificación es una herramienta que el centro puede diseñar para incrementar su eficacia en la consecución de los objetivos, en el caso de que se utilice, hay que tener en cuenta cómo se emplea para alcanzar las metas y mejorar la calidad de la atención a la diversidad.

En este apartado del mencionado instrumento se da respuesta a 19 indicadores acerca de la importancia de cada uno de los elementos para ofrecer una atención a la diversidad de calidad y el grado de cumplimiento en los diferentes centros.

Estimar la atención a la diversidad en los centros educativos supone analizar elementos que tienen que ver con el programa de formación del alumnado ya que tienen que conseguir unos objetivos previamente establecidos con el fin de alcanzar unos conocimientos y destrezas específicas.

El centro educativo es una organización al servicio de un proyecto común. La optimización del proceso educativo está vinculada a la normalización y al desarrollo de centros escolares abiertos y flexibles a las necesidades del alumnado. Debe poseer una selección adecuada y actualizada de la legislación con los aspectos importantes para la calidad de la inte- 
gración. El currículum, por su parte, ha de ser flexible, amplio y equilibrado (Gráfico 5). A partir de la Ley de ordenación general del sistema educativo (LOGSE) encontramos una nueva definición: "conjunto de objetivos, contenidos, métodos pedagógicos y criterios de evaluación de cada uno de los niveles, etapas, ciclos, grados y modalidades del sistema educativo que regula la práctica docente", presenta un carácter abierto y flexible para permitir su adecuación a la realidad del contexto socio-económico y cultural de cada centro escolar, respetar el pluralismo cultural y responder, asimismo, a la diversidad de capacidades, intereses y motivaciones del alumnado implicando activamente al profesorado en su desarrollo y concreción.

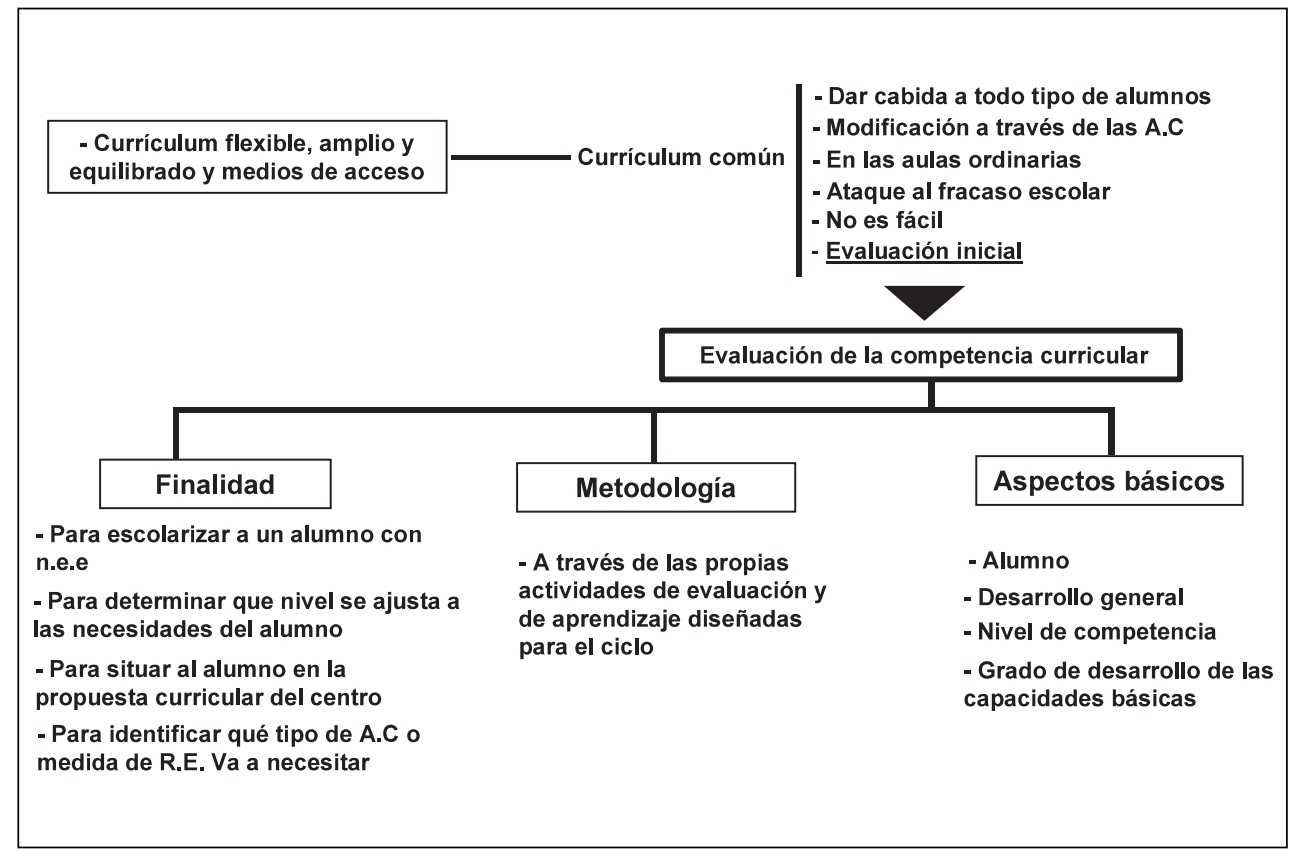

GRÁFICO 5.

Currículum.

Uno de los aspectos fundamentales en un programa de formación es la organización de la enseñanza desde el centro educativo, su distribución y adecuación al tipo de alumnado. Es por eso que debemos atender dentro de esta dimensión a los proyectos que se realizan: el proyecto educativo de centro (PEC) (Gráfico 6), el proyecto curricular de centro (PCC) (Gráfico 7), las programaciones de aula (Gráfico 8), las adaptaciones curriculares y las medidas de refuerzo educativo (Gráfico 9).

Para valorar estos apartados se introdujeron en el instrumento 83 ítems.

Las instalaciones, recursos y los recursos humanos, como reflejamos en el gráfico 10, constituyen la condición de partida con las que cuenta el centro docente para desarrollar su actividad. Son condiciones de entrada que posibilitan su funcionamiento. 


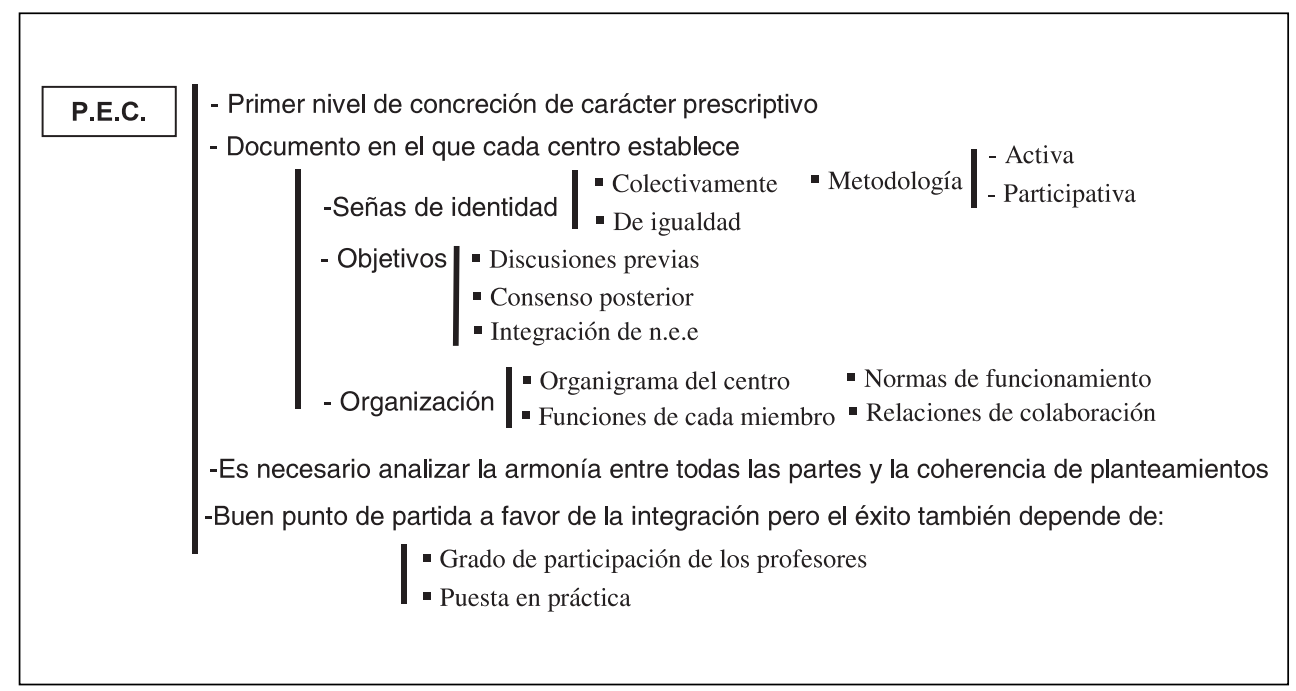

GRÁFICO 6.

Proyecto educativo de centro.

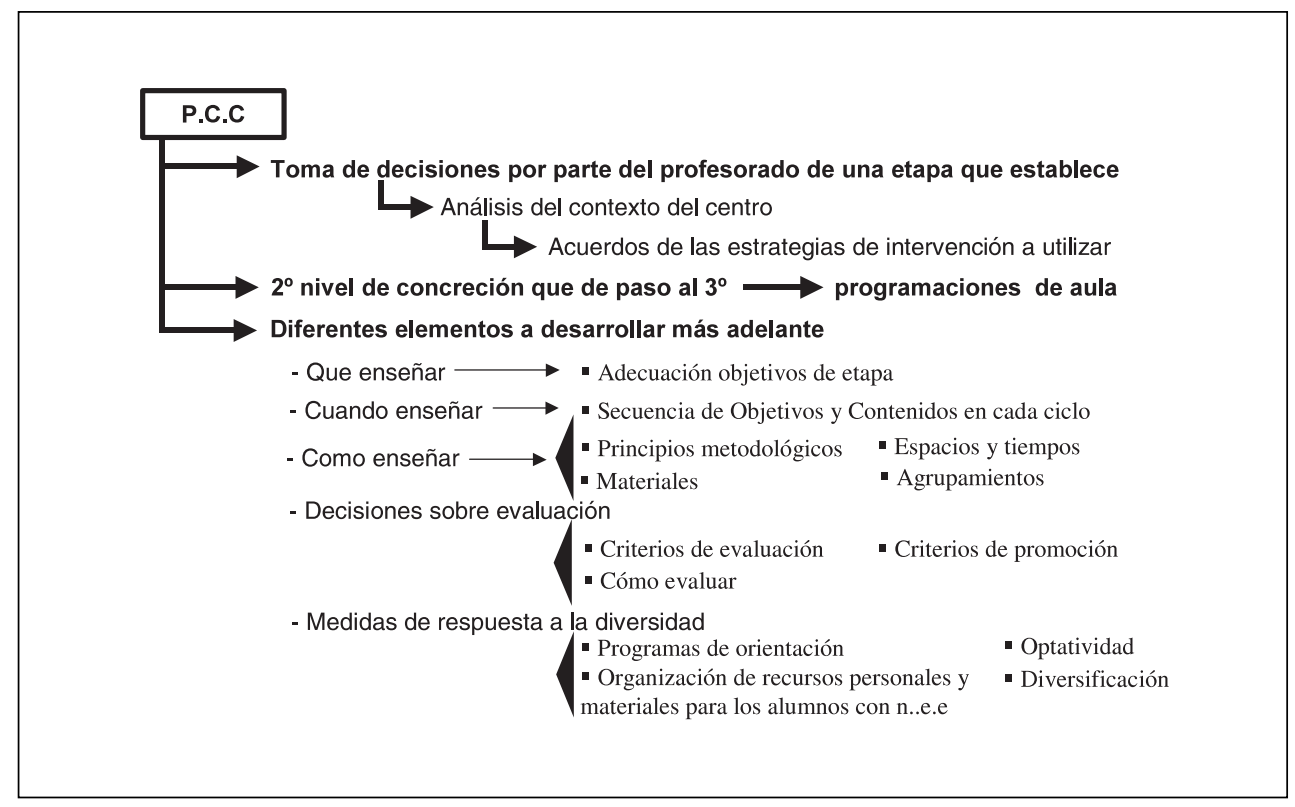

GRÁFICO 7.

Proyecto curricular de centro. 


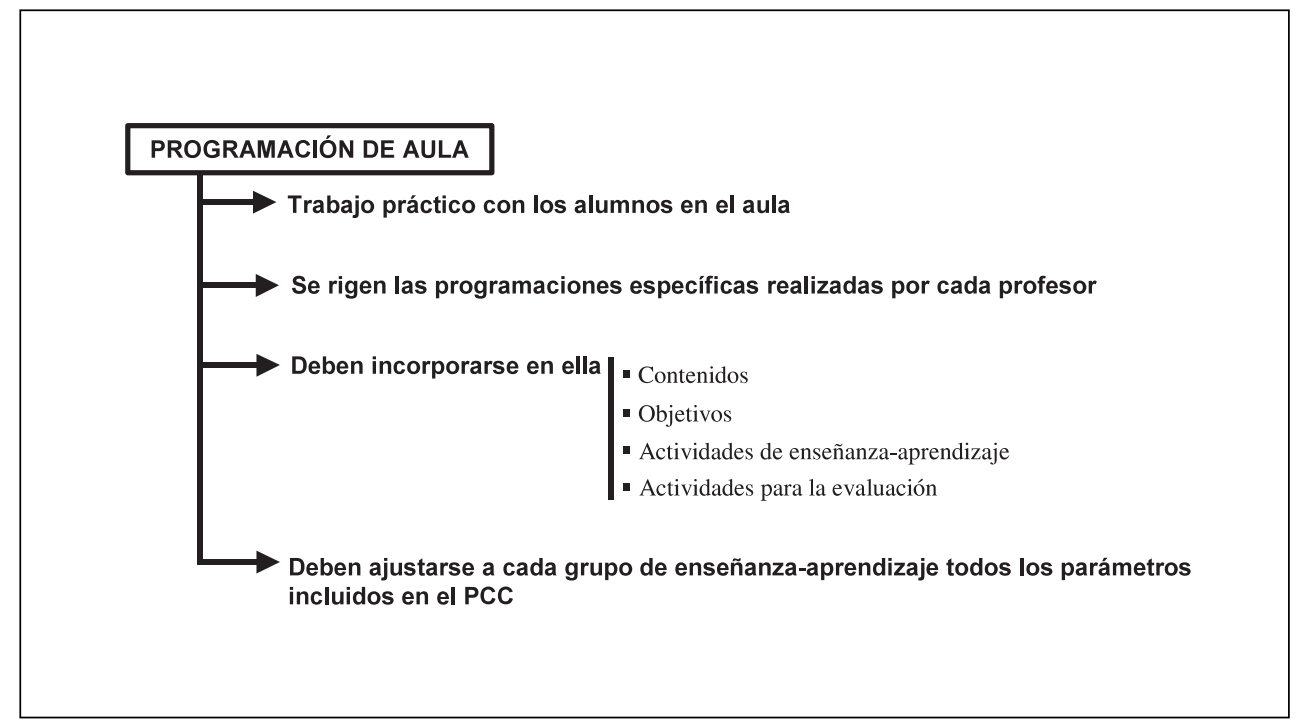

GRÁFICO 8.

Programación de aula.

MEDIDAS DE REFUERZO EDUCATIVO

Medida ordinaria de atención a la diversidad que afecta a los elementos no prescriptivos del currículum - Secuencia de contenidos

- Formas e instrumentos de evaluación

- Organización del aula

- Agrupamientos de los alumnos

- Metodología

Para que sea de calidad

- Es necesario aplicarlo en el propio aula

- No es necesario pasar por la inspección

- Es necesario planteártelo antes de una ACI

GRÁFICO 9.

Medidas de refuerzo educativo. 


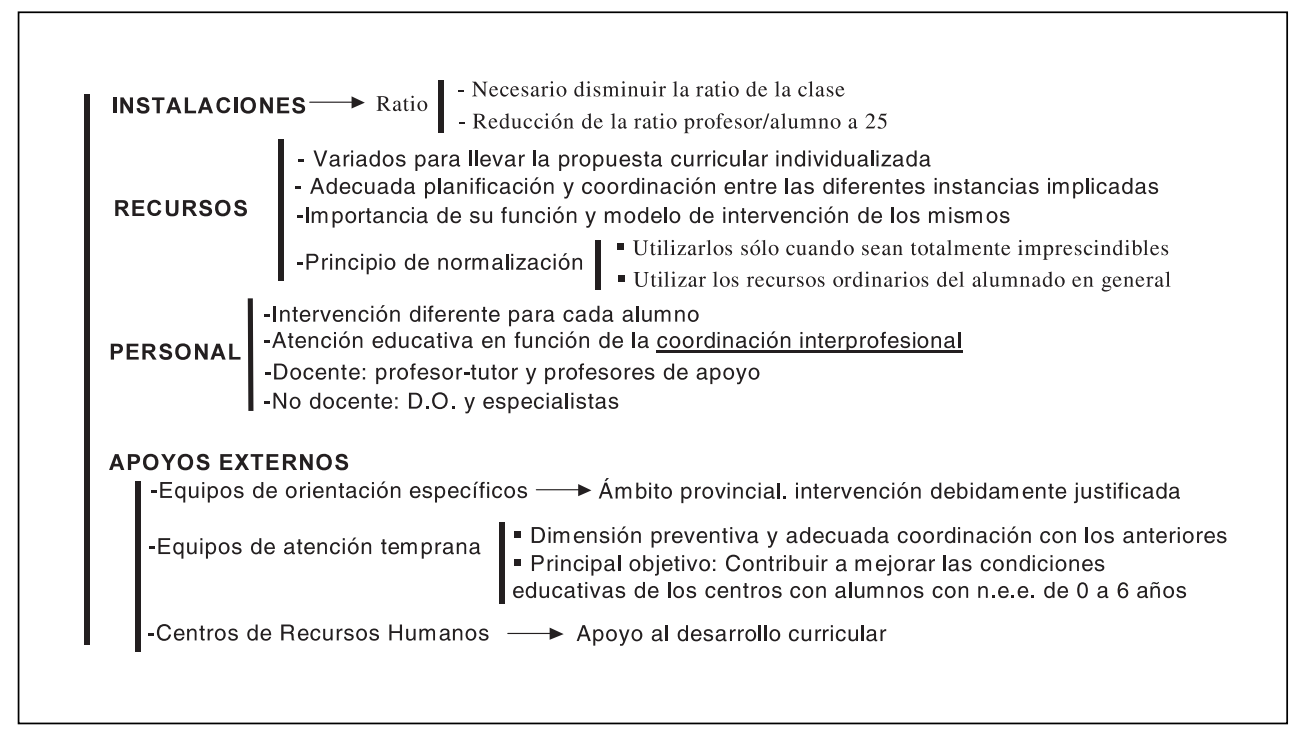

\section{GRÁFICO 10. \\ Instalaciones, recursos y recursos humanos.}

Este apartado, en el instrumento, se divide en dos partes: la primera hace referencia a los recursos humanos y se proponen 49 ítems para valorar. La segunda hace referencia a las instalaciones y recursos, en la cual se proponen 6 ítems para valorar.

Finalmente, se introduce el apartado de resultados en el cual se intentará determinar el grado en que se logra una atención a la diversidad de calidad en todas las dimensiones analizadas hasta el momento, descritas en términos de satisfacción.

En el instrumento, en este apartado, se proponen 23 ítems.

\section{Reflexiones sobre la propuesta de medidas de atención a la diversidad}

Cuando hablamos de diversidad no nos referimos exclusivamente a capacidades sino que incluimos también los intereses, las expectativas, la formación inicial, los estilos de aprendizaje, la procedencia socio-cultural, los estímulos y ayudas de fuera del centro, la personalidad, y dentro de ésta el autoconcepto y muchas otras características del alumnado. Para dar una respuesta a la diversidad es importante la renovación pedagógica que permita atender a la heterogeneidad dentro de las aulas.

En el plan de atención a la diversidad deberán constar cuando menos dos tipos de medidas, las curriculares (Gráfico 11) y las organizativas (Gráfico 12). 


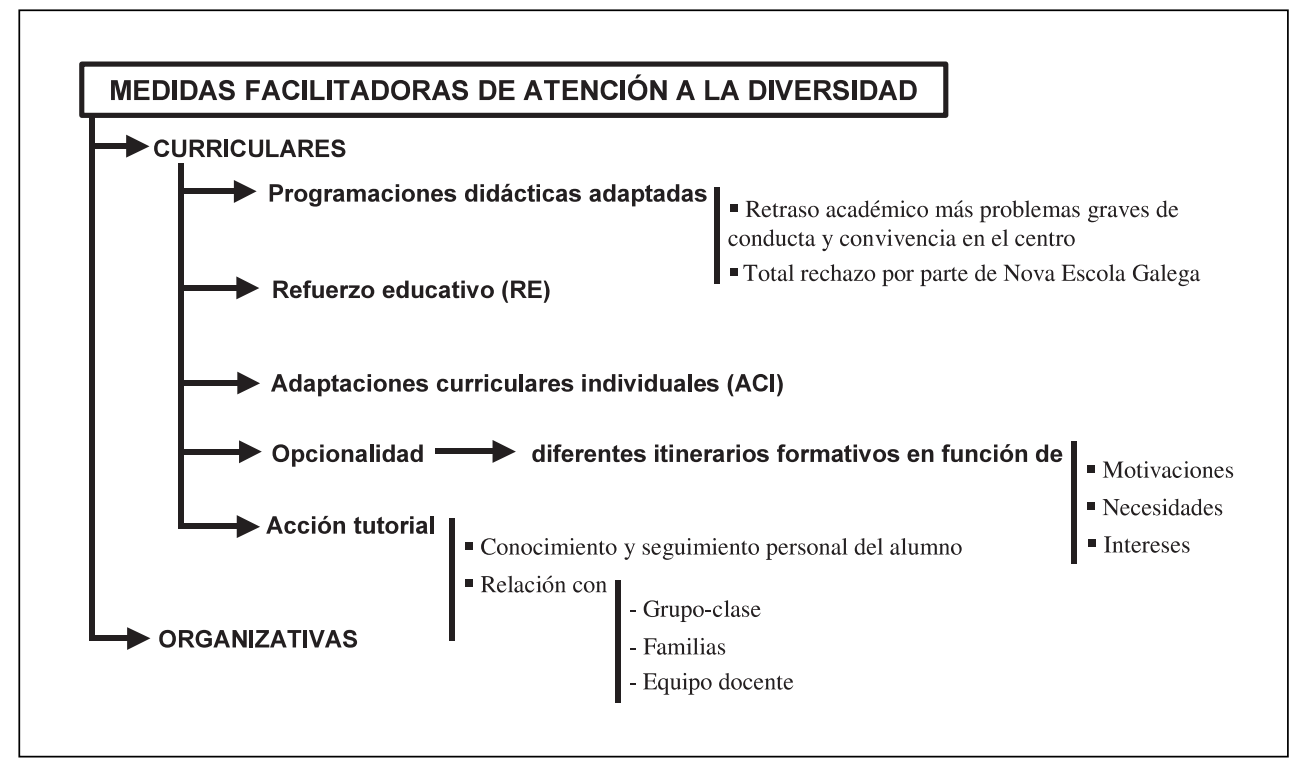

GRÁFICO 11.

Medidas curriculares.

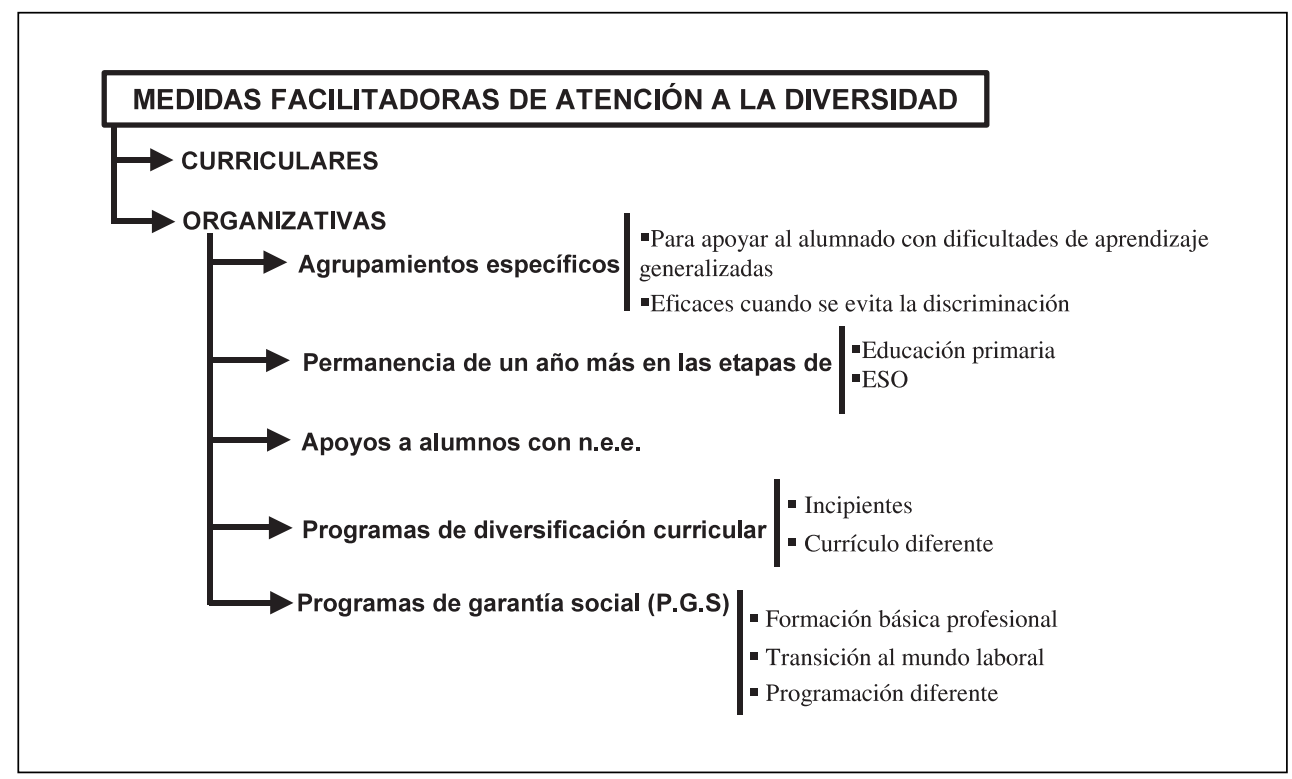

GRÁFICO 12.

Medidas organizativas. 


\section{Referencias bibliográficas}

De la Orden, A. y Hernández, M. J. (1989). "Funcionamiento departamental y recursos físicos y materiales en la Universidad", Revista de Investigación educativa, 7, 13: 7-19.

De la Orden, A. (1995). "Evaluación de la organización de centros escolares". Revista Galega de Psicopedagogía. Monográfico no 6: 51-66.

De Miguel, M.; Madrid, V.; Noriega, J., y Rodríguez, B. (1994). Evaluación para la calidad de los institutos de educación secundaria, Escuela Española, Madrid.

García Pastor, C. (1993). Una escuela común para niños diferentes. E.V., Barcelona.

López Sepúlveda, C. (1988). El refuerzo pedagógico, Escuela Española, Madrid.

Muñoz Cadavid, M. A. (2000). "La observación: una primera aproximación al alumno/a con necesidades educativas en contextos de integración". Revista galego-portuguesa de psicoloxía e educación, 5, 4: 33-41.

\section{Normativa legal}

Constitución Española de 1978 (BOE, 19-12-1978).

Ley 13/1982, de 7 de Abril (BOE, de 30).

RD 334/1985, dc 6 de Marzo (BOE, 16-03-85).

LOGSE (BOE 4.10.90).

Fecha de recepción: 16-01-2004

Fecha de revisión: 19-05-2004

Fecha de aceptación: 05-11-2004 